\title{
Membrane-Based Scanning Force Microscopy
}

\section{Journal Article}

Author(s):

Hälg, David (D); Gisler, Thomas; Tsaturyan, Yeghishe; Catalini, Letizia; Grob, Urs; Krass, Marc-Dominik; Héritier, Martin; Mattiat, Hinrich; Thamm, Ann-Katrin; Schirhagl, Romana; Langman, Eric C.; Schliesser, Albert; Degen, Christian L.; Eichler, Alexander

Publication date:

2021-02

\section{Permanent link:}

https://doi.org/10.3929/ethz-b-000472073

\section{Rights / license:}

In Copyright - Non-Commercial Use Permitted

\section{Originally published in:}

Physical Review Applied 15(2), https://doi.org/10.1103/PhysRevApplied.15.L021001

\section{Funding acknowledgement:}

177198 - Zeptonewton force sensing on a membrane resonator platform (SNF)

309301 - Three-dimensional Magnetic Resonance Imaging at Molecular Resolution (EC)

ETH-03 16-1 - Attempt at the mechanical detection of a single nuclear spin (ETHZ) 


\title{
Membrane-Based Scanning Force Microscopy
}

\author{
David Hälg, ${ }^{1, \ddagger}$ Thomas Gisler, ${ }^{1, \ddagger}$ Yeghishe Tsaturyan, ${ }^{2, \dagger}$ Letizia Catalini $\odot,{ }^{2,3}$ Urs Grob, ${ }^{1}$ \\ Marc-Dominik Krass, ${ }^{1}$ Martin Héritier, ${ }^{1}$ Hinrich Mattiat, ${ }^{4}$ Ann-Katrin Thamm, ${ }^{1}$ Romana Schirhagl, ${ }^{5}$ \\ Eric C. Langman, ${ }^{2,3}$ Albert Schliesser ${ }^{2,3}$ Christian L. Degen, ${ }^{1}$ and Alexander Eichler ${ }^{1,{ }^{*}}$ \\ ${ }^{1}$ Laboratory for Solid State Physics, ETH Zürich, Zürich 8093, Switzerland \\ ${ }^{2}$ Niels Bohr Institute, University of Copenhagen, Copenhagen 2100, Denmark \\ ${ }^{3}$ Center for Hybrid Quantum Networks, Niels Bohr Institute, University of Copenhagen, Copenhagen 2100, \\ Denmark \\ ${ }^{4}$ Department of Physics, University of Basel, Basel 4056, Switzerland \\ ${ }^{5}$ Department of Biomedical Engineering, Groningen University, Groningen 9713 AW, Netherlands
}

(Received 16 October 2020; accepted 23 December 2020; published 5 February 2021)

\begin{abstract}
We report the development of a scanning force microscope based on an ultrasensitive silicon nitride membrane optomechanical transducer. Our development is made possible by inverting the standard microscope geometry - in our instrument, the substrate is vibrating and the scanning tip is at rest. We present topography images of samples placed on the membrane surface. Our measurements demonstrate that the membrane retains an excellent force sensitivity when loaded with samples and in the presence of a scanning tip. We discuss the prospects and limitations of our instrument as a quantum-limited force sensor and imaging tool.
\end{abstract}

DOI: 10.1103/PhysRevApplied.15.L021001

The coupling between optical and mechanical degrees of freedom plays a fundamental role in physics and forms the basis for many important applications in metrology and signal transduction [1]. The field of cavity optomechanics, in particular, has enabled the detection of vibrations with very high sensitivity $[2,3]$ extending to below the standard quantum limit [4], demonstrated squeezed phononic states [5-7] and quantum ground-state cooling of "massive" mechanical resonators [8-12], and enabled optoelectromechanical signal transduction with quantumlimited efficiency $[13,14]$. Such advances raise the question whether optomechanical systems could be harnessed for quantum-limited force sensors and scanning force microscopes [15].

Sensitive force microscopy has important applications for the imaging of isolated electronic charges $[16,17]$ and spins [18], nanoscale nuclear spin density distributions [19, 20], as well as magnetic fields generated by nanoscale currents [21]. A long-standing goal is the detection of a single nuclear spin, which would constitute a milestone towards atomic-resolution magnetic resonance imaging [20,2226]. This vision hinges crucially on the development of mechanical transducers that combine exceptionally low

\footnotetext{
*eichlera@ethz.ch

${ }^{\dagger}$ Present address: Pritzker School of Molecular Engineering, University of Chicago, Chicago, Illinois 60637, USA.

$\$$ These authors contributed equally to this work.
}

thermomechanical force noise with very low detector noise. In recent years, impressive progress in both respects has been achieved with silicon nitride $\left(\mathrm{Si}_{3} \mathrm{~N}_{4}\right)$ strings and membranes, whose high strain, extreme aspect ratio, and phononic crystal clamp engineering have resulted in very high quality factors [27-37]. Membranes and string resonators, however, require clamping on opposite ends and can therefore not be used in the cantilever or pendulum geometry adopted in ultrasensitive scanning force microscopy experiments [38,39]. This obstacle has so far impeded the implementation of membranes in nanoscale scanning force microscopy.

In this work, we demonstrate a scanning force microscope based on a silicon nitride membrane sensor. We accommodate the seemingly incompatible geometries by inverting the standard microscope paradigm; in our setup, the sample plate is also the force sensor, which we approach with a static (nonvibrating) scanning tip. We avoid snap-in-to contact by employing high-stiffness membranes, and achieve high force sensitivity through the use of state-of-the-art phononic bandgap membranes [35] with very high quality factors $\left(Q=10^{7}-10^{8}\right)$. At the same time, the extended membrane surface allows placement of a wide range of samples without significant reduction of $Q$ (see the Supplemental Material [41]). As a proof of principle, we demonstrate scanning probe operation by imaging the nanometer-resolution topography of gold nanospheres and tobacco mosaic virus (TMV) particles deposited on the membrane surface. When combined with a precise optical 
cavity readout and specialized spin-mechanics coupling protocols [40] at cryogenic temperatures, our approach is expected to enable quantum-limited scanning force microscopy with the capability to detect individual nuclear spins.

Our inverted scanning force microscope consists of a static tip scanning above a vibrating silicon nitride membrane (Fig. 1). The membrane oscillates in the out-of-plane direction, unlike other ultrasensitive force microscopes that use a pendulum geometry [42]. The membrane simultaneously acts as the sample platform and the optomechanical force transducer. To improve the quality factor and hence the force sensitivity, we use membranes that have been structured with a hexagonal lattice of perforations to create a bandgap for out-of-plane oscillations. At the center of the membrane sits a larger (approximately $100 \mu \mathrm{m}$ ) unperforated area. This "defect" is capable of supporting localized mechanical modes with eigenfrequencies inside the bandgap and mechanical quality factors up to $1 \times 10^{9}$ $[35,36]$. Similarly high quality factors have been reported

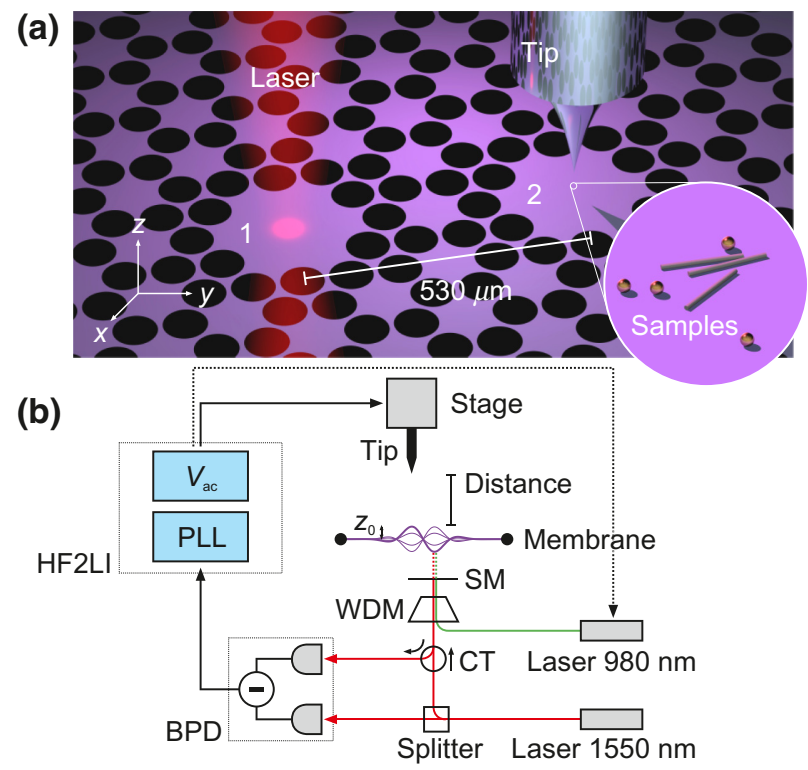

FIG. 1. (a) We use a 41-nm-thick silicon nitride membrane as the nanomechanical force sensor. A hole pattern acts as a phonon shield, while two coupled "defect" areas (labeled 1 and 2) undergo out-of-plane vibrations. We load gold nanoparticles and tobacco mosaic viruses onto one of the defect areas and measure the vibrations on the second one with a laser interferometer. The scanning force microscope is operated in high vacuum to avoid viscous damping. Figure adapted from Ref. [40]. (b) A $1550 \mathrm{~nm}$ laser is used to detect the membrane motion and a $980 \mathrm{~nm}$ laser drives the membrane via radiation-pressure forces. Membrane vibrations are detected with a lock-in amplifier (HF2LI). The ac output of the lock-in amplifier is used to modulate the potential of the scanning tip or the power of the driving laser. CT is the circulator, WDM is the wavelength-division multiplexer, SM is the semitransparent mirror, BPD is the balanced photodetector, and PLL is the phase-locked loop. for other types of perforations [43] and for geometries with narrow tethers [33].

Our membranes have a pair of defects that are separated by a distance of a few hundred micrometers. The defects are mechanically coupled and form hybridized normal modes - symmetric and antisymmetric - with frequencies that are a few tens of kilohertz apart [see Fig. 1(a)] [44]. In future experiments, we expect to use the double-mode structures for parametric up-conversion of magnetic forces from nuclear spins [40]. Here, we take advantage of the fact that the two defects allow us to spatially separate the sites for sample placement and for optical readout.

We detect the membrane vibrations with a fiberbased laser interferometer positioned below the membrane [Fig. 1(b)] [45]. The two arms of the interferometer are formed by a semitransparent mirror at the fiber end and by the membrane itself. Because of the low membrane reflectivity (approximately 6\%) we employ a balanced photodetector that offsets the large reflection from the reference arm. The interferometer has a double-sided power spectral density noise floor of approximately $10^{-28} \mathrm{~m}^{2} / \mathrm{Hz}$. We drive the membrane either via the radiation pressure force of a second driving laser or through an electrostatic modulation of the scanning tip [see Fig. 1(b)]. We detect the signal by a lock-in amplifier, and utilize the amplifier's on-board phase-locked loop to maintain resonant driving of membrane modes in the presence of thermal frequency drifts.

To realize the scanning probe functionality, we integrate a scanning tip on an $x y z$ piezo translation stage above the membrane. We use metal-coated silicon tips from commercial atomic force microscope (AFM) cantilevers that are glued to a micromachined metallic needle for rigid support. The metal finish of the scanning tip is convenient for applying a tip potential and for implementing a dynamic electrostatic driving. We have also tested scanning tunneling microscopy tips made from tungsten by wet etching, but found them to degrade rapidly due to the high stiffness of the membrane.

To characterize a membrane, we first sweep the frequency $f_{d}$ of a small force to drive vibrations in a spectral range of interest. The frequency spectrum shows a dense quasicontinuum of modes that is interrupted by a gap from approximately 1.36 to $1.65 \mathrm{MHz}$ [Fig. 2(a)]. Inside the gap, we find only a few out-of-plane modes that typically feature quality factors $Q>10^{7}$ [35]. We identify the doubledefect modes by the pair of resonances around $1.4 \mathrm{MHz}$ whose frequency splitting agrees with that expected from theory [44]. In the following, we focus on the antisymmetric mode with a resonance frequency of $f_{0}=1.417 \mathrm{MHz}$. We determine the precise quality factor of the modes via the thermomechanical displacement noise spectrum and through ring-down measurements [Fig. 2(b)].

To engage the tip, we approach the surface of the membrane until we observe a shift of the resonance frequency 
(a)

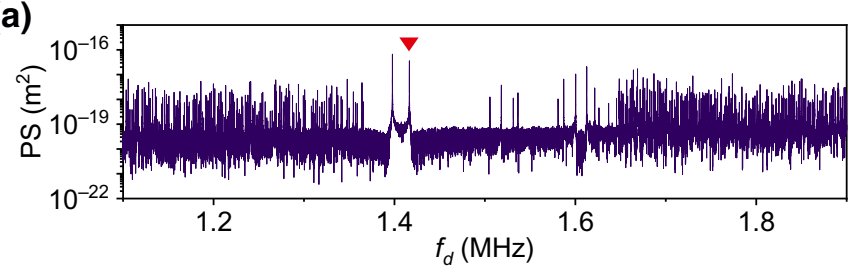

(b)

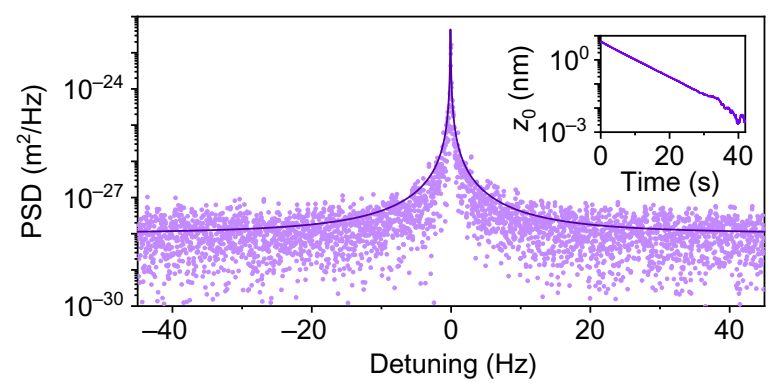

(c)

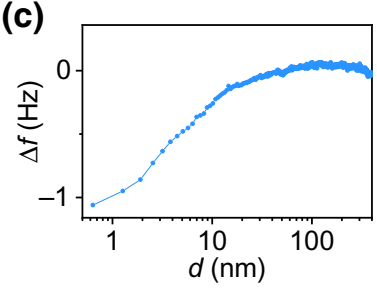

(e) 30

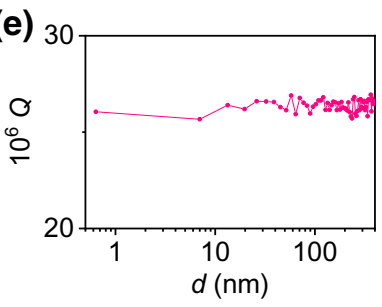

(d)

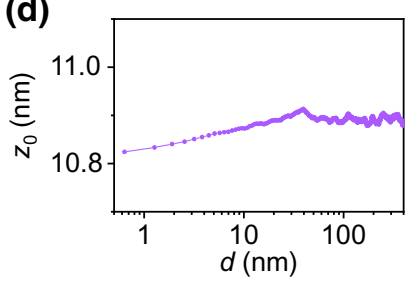

(f)

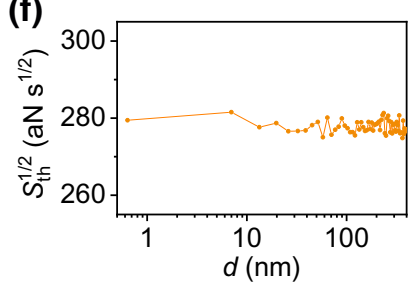

FIG. 2. (a) Displacement power spectrum (PS) obtained by slowly sweeping $f_{d}$ applied through the modulated tip voltage. The red arrow marks the high- $Q$ mode used in this work. The force is applied through the modulated potential of the scanning tip far away from the surface. (b) Thermal displacement power spectral density (PSD) of the high- $Q$ mode. The solid line is a fit to the model of a harmonic resonator. The value $Q=2.6 \times 10^{7}$ is independently obtained from a ringdown measurement (inset) and agrees well with the peak width. The spring constant is $k=m\left(2 \pi f_{0}\right)^{2}=1100 \mathrm{~N} / \mathrm{m}$. (c)-(e) Shift of $f_{0}$, vibration amplitude, and $Q$ measured as a function of tip-surface distance $d$, respectively. The membrane is driven by a radiation pressure force with a constant amplitude. We define the touch position $(d=0)$ from threshold values where $\Delta f$ and $z_{0}$ exceed a set value. (For example, $\Delta f=0.3 \mathrm{~Hz}$ and $\Delta z_{0}=30 \%$ for the scans shown in this paper.) (f) The single-sided thermomechanical force noise $S_{\mathrm{th}}^{1 / 2}=4 k_{B} T \gamma_{0}$ calculated from (c)-(e) and the fit results from (b). All measurements in the main manuscript are from a single membrane device. Data from additional devices are shown in the Supplemental Material [41].

$\Delta f$ as well as a reduction of the amplitude $z_{0}$ [see Figs. 2(c) and 2(d)]. We find that the frequency and amplitude only change at very close approach, $d<10 \mathrm{~nm}$. This behavior is expected because the membrane has a high stiffness $k \sim 10^{3} \mathrm{~N} / \mathrm{m}$. We further measure $Q$ as a function of $d$, and observe no noticeable decrease even at very close approach [Fig. 2(e)]. This observation is important, because it indicates little noncontact friction (NCF) $[46,47]$ and little loss in force sensitivity in close proximity to the membrane. Shown in Fig. 2(f), the single-sided thermomechanical force noise, defined by $S_{\mathrm{th}}^{1 / 2}=4 k_{B} T \gamma_{0}$, is approximately $280 \mathrm{aN} / \sqrt{\mathrm{Hz}}$ at a distance of $20 \mathrm{~nm}$ and changes by less than $1 \%$ when lowering the distance to $1 \mathrm{~nm}$. Here, $T \approx 300 \mathrm{~K}$ is the ambient temperature, $k_{B}$ is Boltzmann's constant, and $\gamma_{0}=2 \pi f_{0} m / Q$ is the damping coefficient when the scanning tip is far from the surface, with $m=14 \mathrm{ng}$ the effective mass. The damping added by $\mathrm{NCF}$ at $d=1 \mathrm{~nm}$ thus only amounts to $\gamma_{\mathrm{nc}} \lesssim 0.01 \gamma_{0} \approx$ $5.5 \times 10^{-14} \mathrm{~kg} / \mathrm{s}$. This value compares favorably to $\gamma_{\mathrm{nc}} \approx$ $1.5 \times 10^{-13} \mathrm{~kg} / \mathrm{s}$ and $10^{-12} \mathrm{~kg} / \mathrm{s}$ found at room temperature for a pendulum-style cantilever over a gold surface at distances of 10 and $2 \mathrm{~nm}$, respectively [48]. The low NCF of our membrane sensor is probably due to a combination of (i) reduced electrical fluctuations at megahertz frequencies relative to the kilohertz range $[49,50]$, and (ii) smaller interaction between tip and surface for out-of-plane oscillations compared to the shear motion in the pendulum geometry. We expect the NCF to be further reduced at cryogenic temperatures $[48,51]$.

We demonstrate scanning force microscopy by imaging the topography of samples dispersed on the membrane surface. Because of the high stiffness of the membrane, we build up images by measuring the "touch point" at each pixel, rather than by regulating the frequency or amplitude via distance feedback. We define the touch position from threshold values of $\Delta f$ and $z_{0}$, that is, we terminate the approach (and define a "touch") when the change in either frequency or amplitude exceeds a set value. As shown below, this procedure is reproducible with subnanometer accuracy. In Fig. 3(a), we show a topographic image of gold nanospheres and TMV particles that are deposited from liquid suspension (see the Supplemental Material [41]). Round and elongated objects are clearly visible and correspond in height and length roughly to the expected dimensions of the gold and TMV particles, respectively. Enlarged scans show the same smaller area, once with the membrane driven by an electrostatic force applied through the tip [Fig. 3(b)], and once by radiation pressure driving [Fig. 3(c)]. The two images demonstrate that both actuation methods are well suited for scanning imaging.

In order to evaluate the spatial resolution, we extract linescans in the $x$ direction at different $y$ positions [Fig. 3(d)]. The step size is $13 \mathrm{~nm}$ in these scans, and we find dips between objects that narrow down to a single 


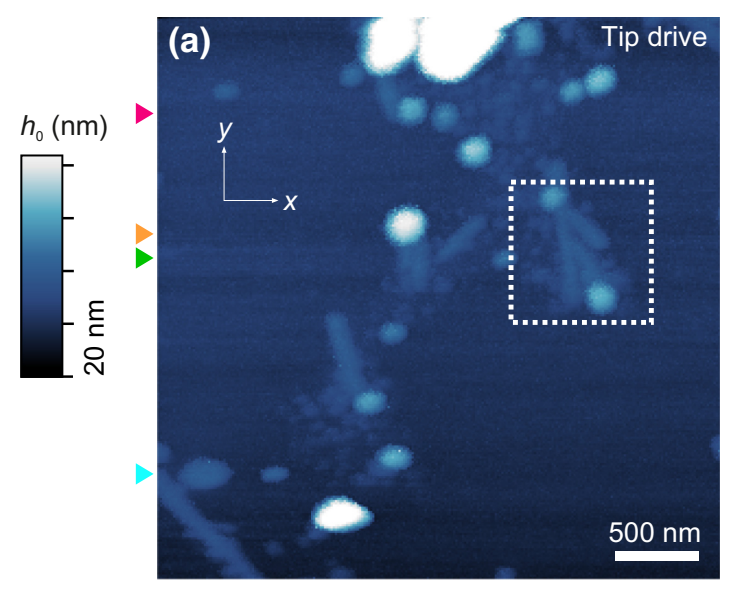

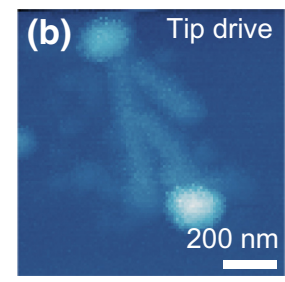

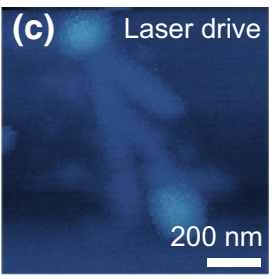

(d)

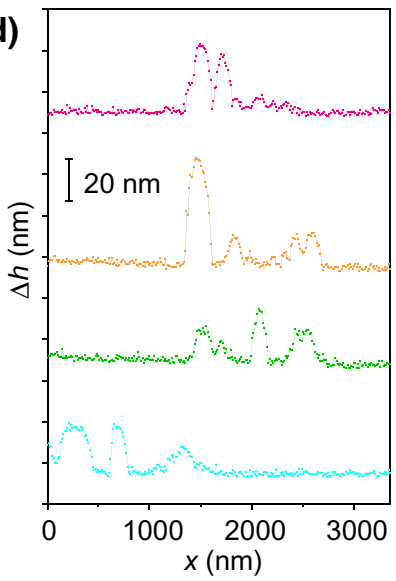

FIG. 3. The relative surface position $h_{0}$ is indicated by the same color bar for all images, with ticks separated by $20 \mathrm{~nm}$. (a) Touch map of the membrane surface with gold nanoparticles (nominal average diameter of $50 \mathrm{~nm}$ ) and TMV samples (diameter of $18 \mathrm{~nm}$, used for $z$-scanner calibration [52]). Step size is $13 \mathrm{~nm}$. Marks on the left-hand side indicate the positions of the linescans in (d). The membrane is driven by modulating the tip voltage. (b) High-resolution scan over the area indicated by a square in (a). Step size is $10 \mathrm{~nm}$. (c) Same scan performed using radiation pressure driving. (d) Linescans extracted from the data in (a). Scans are vertically offset for clarity.

point. From the flat part of the lowest linescan between 2000 and $3360 \mathrm{~nm}$, we obtain a standard deviation in the $z$ direction of $0.3 \mathrm{~nm}$. This value is an upper bound on the resolution in height, as we cannot exclude that some fluctuations stem from real features. The lateral resolution is lower due to convolution with the rounded AFM tip. Given that the cylindrical TMV features in our image have lateral widths of approximately $100 \mathrm{~nm}$, we estimate that the scanning tip has a nominal radius of about $40 \mathrm{~nm}$. If desired, this resolution could be improved by integrating high-resolution AFM probes.

Our touch map imaging method currently is very slow. Each pixel requires roughly $8 \mathrm{~s}$ of acquisition time, and the measurement of a large and detailed image, such as that shown in Fig. 3(a) with $256 \times 256$ pixels, requires several days. This limitation is probably due to the fact that changes in the membranes response can often only be detected at a distance of a few nanometers because of the high mechanical stiffness. In conjunction with the limited precision of our $z$ scanner, the stiffness makes the use of standard $z$ feedback challenging. We expect that this issue will be alleviated for membranes with a lower stiffness in the 1-10 N/m range (see the Supplemental Material [41]).

The main prospect of optomechanical membrane sensors is the quantum-limited, subattonewton detection of weak forces, rather than topographic imaging. We conclude our paper by discussing the challenges and key steps towards reaching this goal. The membrane used in this work has an intrinsic single-sided room-temperature thermomechanical force noise of $S_{\mathrm{th}}^{1 / 2}=280 \mathrm{aN} \mathrm{Hz}^{-1 / 2}$ [Fig. 2(f)]. This figure is not yet competitive with stateof-the-art cantilevers [42], carbon nanotubes [53,54], or nanowires [51,55-57] that reached a force noise between 10 and $100 \mathrm{aN} \mathrm{Hz}^{-1 / 2}$ at room temperature and less than
$1 \mathrm{aN} \mathrm{Hz}^{-1 / 2}$ at cryogenic temperatures. The best membrane devices we tested in a setup without a scanning stage featured $S_{\mathrm{th}}{ }^{1 / 2}=37 \mathrm{aN} \mathrm{Hz}^{-1 / 2}$ [44], which is close to the $S_{\mathrm{th}}{ }^{1 / 2}=19.5 \mathrm{aN} \mathrm{Hz}^{-1 / 2}$ reported at room temperature for trampoline geometries [33].

Looking forward, we expect to improve the sensitivity by engineering central defects with lower mode masses and higher $Q$, as well as by moving to cryogenic operation. A prospective device will have $m=77 \mathrm{pg}, k=3 \mathrm{~N} / \mathrm{m}$, $f_{0}=1 \mathrm{MHz}$, and a room temperature $Q$ of about $1.4 \times 10^{8}$, corresponding to $S_{\mathrm{th}}{ }^{1 / 2}=7.6 \mathrm{aN} \mathrm{Hz}^{-1 / 2}$. When cooled to $T=0.2 \mathrm{~K}$, we expect $Q=4.8 \times 10^{8}$ and $S_{\mathrm{th}}{ }^{1 / 2}=$ $0.1 \mathrm{aN} \mathrm{Hz}^{-1 / 2}$ (see the Supplemental Material [41]), which would be sufficient to detect the magnetic moment of a single proton [58].

An important obstacle for cryogenic detection is the limited displacement sensitivity of our present optical interferometer. The noise in the detected photocurrent amounts to an imprecision in the membrane position measurement of the order $S_{\mathrm{det}} \sim 10^{-28} \mathrm{~m}^{2} / \mathrm{Hz}$. When cooled to $T=0.2 \mathrm{~K}$, the thermal motion of the resonator shown in Fig. 2 is only $z_{\mathrm{rms}}=5 \times 10^{-14} \mathrm{~m}$. Allowing for some detection bandwidth of the order of tens of hertz, a displacement sensitivity $S_{\text {det }} \lesssim 10^{-30} \mathrm{~m}^{2} / \mathrm{Hz}$ will be needed (see the Supplemental Material [41]). An even lower detector noise is required to implement active feedback cooling of the membrane mode, which can be useful to control the mechanical response time and thermal motion amplitude [19,36]. A future version of our inverted scanning microscope will therefore incorporate an optical readout cavity in a membrane-inthe-middle geometry [59]. A comparably low finesse of $\mathcal{F} \approx 500$ will be sufficient to achieve a sensitivity below $10^{-30} \mathrm{~m}^{2} / \mathrm{Hz}$. 
When implementing such optical detection on mechanical sensors with very low thermomechanical force noise, it can become necessary to take the quantum backaction of the optical measurement into account [2-4]. This backaction appears as an additional force noise $S_{\mathrm{qba}}$, produced by the shot noise of the radiation pressure acting on the membrane. It is bound by a Heisenberg-type relation to $S_{\mathrm{qba}} \geq \hbar^{2} / S_{\text {det }}$, where $\hbar$ is the reduced Planck constant. For the prospective membrane described above, one reaches a backaction-dominated regime with $S_{\mathrm{qba}} \geq$ $S_{\text {th }}$ for $S_{\text {det }} \approx 10^{-30} \mathrm{~m}^{2} / \mathrm{Hz}$. Eventually, one could use advanced quantum measurement protocols to evade quantum backaction $[4,60]$.

From our measurements in Figs. 2(d)-2(f), we conclude that NCF is not a critical obstacle for reaching sub-aN force sensitivity. The NCF observed at $d=1 \mathrm{~nm}\left(\gamma_{\mathrm{nc}}=\right.$ $5.5 \times 10^{-14} \mathrm{~kg} / \mathrm{s}$ ) corresponds to a thermal force noise of $S_{\text {th }}^{1 / 2}=0.8 \mathrm{aN} \mathrm{Hz}^{-1 / 2}$ at $T=0.2 \mathrm{~K}$. We expect that the $\mathrm{NCF}$ will be further reduced at cryogenic temperatures and larger stand-off distances. For example, Stipe et al. [48] found a reduction in $\gamma_{\mathrm{nc}}$ by a factor of 6 times when lowering the temperature from 295 to $77 \mathrm{~K}$ and a reduction proportional to $d^{-1.3}$ with distance for a pendulum cantilever. An even stronger reduction proportional to $d^{-3}$ was reported for a parallel cantilever orientation [61]. These results suggest that NCF will be negligible for distances larger than a few nanometers.

In some cases, specialized measurement protocols are required to make use of mechanical sensors in the megahertz range. We have recently devised a parametric upconversion protocol [40], based on previous work [62,63], to make megahertz sensors compatible with the requirements of magnetic resonance force microscopy [20]. For our concrete protocol and for current device parameters, we predict that nonlinear damping limits the force sensitivity to about $0.2 \mathrm{aN}^{2} / \mathrm{Hz}$ [40].

In summary, we demonstrate an inverted approach to scanning probe microscopy based on a vibrating silicon nitride membrane. Our platform offers excellent sensitivity, approximately $280 \mathrm{aN} \mathrm{Hz}^{-1 / 2}$ at room temperature, and a prospect for significant future improvement. The force sensitivity remains essentially unchanged down to tip-surface separations of $d \sim 1 \mathrm{~nm}$. We demonstrate topographic imaging of gold nanospheres and TMV particles dispersed on the membrane surface.

The low force noise and compatibility of membranes with high-finesse optical cavities are expected to permit quantum-limited force detection. At sub-Kelvin temperatures, prospective devices will have dissipation-limited quantum coherence times of the order of $\tau_{\text {coh }}=\hbar Q / k_{B} T \approx$ $17 \mathrm{~ms}$, much longer than any other scanning force probe demonstrated to date, as well as force sensitivities around $0.1 \mathrm{aN} \mathrm{Hz}^{-1 / 2}$. Important applications of ultrasensitive, quantum-limited force microscopy include the investigation of single spins [18], atomic-scale nuclear magnetic resonance [20,22], electrical quantum dots [64], or skyrmions [65], as well as strong spin-mechanics coupling in quantum hybrid devices.

Acknowledgments. We gratefully acknowledge technical support by the mechanical workshop and the engineering office at ETH Zurich, and thank J. Chen for providing data of quantum-limited optomechanical measurements. This work is supported by the Swiss National Science Foundation (SNFS) through the National Center of Competence in Research in Quantum Science and Technology (NCCR QSIT), the Sinergia grant (CRSII5_177198/1), and the project Grant No. 200020-178863, Danmarks Grundforskningsfond (DNRF) Center of Excellence Hy-Q, the European Research Council through the ERC Starting Grants "NANOMRI" (grant agreement 309301), "QCEOM" (grant agreement 638765), ERC proof-of-concept grant "ULTRAFORS" (grant agreement 825797), and a Marie-Curie Fellowship "Nano-MRI" (325866), as well as an ETH research grant (ETH-03 16-1).

[1] M. Aspelmeyer, T. J. Kippenberg, and F. Marquardt, Rev. Mod. Phys. 86, 1391 (2014).

[2] J. D. Teufel, T. Donner, M. A. Castellanos-Beltran, J. W. Harlow, and K. W. Lehnert, Nat. Nanotechnol. 4, 820 (2009).

[3] G. Anetsberger, O. Arcizet, Q. P. Unterreithmeier, R. Rivière, A. Schliesser, E. M. Weig, J. P. Kotthaus, and T. J. Kippenberg, Nat. Phys. 5, 909 (2009).

[4] D. Mason, J. Chen, M. Rossi, Y. Tsaturyan, and A. Schliesser, Nat. Phys. 15, 745 (2019).

[5] E. E. Wollman, C. U. Lei, A. J. Weinstein, J. Suh, A. Kronwald, F. Marquardt, A. A. Clerk, and K. C. Schwab, Science 349, 952 (2015).

[6] J.-M. Pirkkalainen, E. Damskägg, M. Brandt, F. Massel, and M. A. Sillanpää, Phys. Rev. Lett. 115, 243601 (2015).

[7] F. Lecocq, J. B. Clark, R. W. Simmonds, J. Aumentado, and J. D. Teufel, Phys. Rev. X 5, 041037 (2015).

[8] I. Wilson-Rae, P. Zoller, and A. İmamoğlu, Phys. Rev. Lett. 92, 075507 (2004).

[9] I. Wilson-Rae, N. Nooshi, W. Zwerger, and T. J. Kippenberg, Phys. Rev. Lett. 99, 093901 (2007).

[10] J. D. Teufel, T. Donner, D. Li, J. W. Harlow, M. S. Allman, K. Cicak, A. J. Sirois, J. D. Whittaker, K. W. Lehnert, and R. W. Simmonds, Nature 475, 359 (2011).

[11] J. Chan, T. P. M. Alegre, A. H. Safavi-Naeini, J. T. Hill, A. Krause, S. Gröblacher, M. Aspelmeyer, and O. Painter, Nature 478, 89 (2011).

[12] R. W. Peterson, T. P. Purdy, N. S. Kampel, R. W. Andrews, P.-L. Yu, K. W. Lehnert, and C. A. Regal, Phys. Rev. Lett. 116, 063601 (2016).

[13] T. Bagci, A. Simonsen, S. Schmid, L. G. Villanueva, E. Zeuthen, J. Appel, J. M. Taylor, A. Sørensen, K. Usami, A. Schliesser, and E. S. Polzik, Nature 507, 81 (2014).

[14] R. W. Andrews, R. W. Peterson, T. P. Purdy, K. Cicak, R. W. Simmonds, C. A. Regal, and K. W. Lehnert, Nat. Phys. 10, 321 (2014). 
[15] G. J. Milburn, K. Jacobs, and D. F. Walls, Phys. Rev. A 50, 5256 (1994).

[16] L. J. Klein and C. C. Williams, Appl. Phys. Lett. 79, 1828 (2001).

[17] R. Stomp, Y. Miyahara, S. Schaer, Q. Sun, H. Guo, P. Grutter, S. Studenikin, P. Poole, and A. Sachrajda, Phys. Rev. Lett. 94, 056802 (2005).

[18] D. Rugar, R. Budakian, H. J. Mamin, and B. W. Chui, Nature 430, 329 (2004).

[19] M. Poggio, C. L. Degen, H. J. Mamin, and D. Rugar, Phys. Rev. Lett. 99, 017201 (2007).

[20] C. L. Degen, M. Poggio, H. J. Mamin, C. T. Rettner, and D. Rugar, Proc. Natl. Acad. Sci. USA 106, 1313 (2009).

[21] Y. Ando, H. Kameda, H. Kubota, and T. Miyazaki, J. Appl. Phys. 87, 5206 (2000).

[22] J. A. Sidles, Appl. Phys. Lett. 58, 2854 (1991).

[23] H. J. Mamin, M. Kim, M. H. Sherwood, C. T. Rettner, K. Ohno, D. D. Awschalom, and D. Rugar, Science 339, 557 (2013).

[24] T. Staudacher, F. Shi, S. Pezzagna, J. Meijer, J. Du, C. A. Meriles, F. Reinhard, and J. Wrachtrup, Science 339, 561 (2013).

[25] M. Loretz, S. Pezzagna, J. Meijer, and C. L. Degen, Appl. Phys. Lett. 104, 033102 (2014).

[26] U. Grob, M.-D. Krass, M. Héritier, R. Pachlatko, J. Rhensius, J. Košata, B. A. J. Moores, H. Takahashi, A. Eichler, and C. L. Degen, Nano Lett. 19, 7935 (2019).

[27] S. S. Verbridge, H. G. Craighead, and J. M. Parpia, Appl. Phys. Lett. 92, 013112 (2008).

[28] B. M. Zwickl, W. E. Shanks, A. M. Jayich, C. Yang, A. C. B. Jayich, J. D. Thompson, and J. G. E. Harris, Appl. Phys. Lett. 92, 103125 (2008).

[29] Q. P. Unterreithmeier, T. Faust, and J. P. Kotthaus, Phys. Rev. Lett. 105, 027205 (2010).

[30] S. Schmid, K. D. Jensen, K. H. Nielsen, and A. Boisen, Phys. Rev. B 84, 165307 (2011).

[31] P.-L. Yu, T. P. Purdy, and C. A. Regal, Phys. Rev. Lett. 108, 083603 (2012).

[32] S. Chakram, Y. S. Patil, L. Chang, and M. Vengalattore, Phys. Rev. Lett. 112, 127201 (2014).

[33] C. Reinhardt, T. Müller, A. Bourassa, and J. C. Sankey, Phys. Rev. X 6, 021001 (2016).

[34] R. A. Norte, J. P. Moura, and S. Gröblacher, Phys. Rev. Lett. 116, 147202 (2016).

[35] Y. Tsaturyan, A. Barg, E. S. Polzik, and A. Schliesser, Nat. Nanotechnol. 12, 776 (2017).

[36] M. Rossi, D. Mason, J. Chen, Y. Tsaturyan, and A. Schliesser, Nature 563, 53 (2018).

[37] A. H. Ghadimi, S. A. Fedorov, N. J. Engelsen, M. J. Bereyhi, R. Schilling, D. J. Wilson, and T. J. Kippenberg, Science 360, 764 (2018).

[38] N. Scozzaro, W. Ruchotzke, A. Belding, J. Cardellino, E. Blomberg, B. McCullian, V. Bhallamudi, D. Pelekhov, and P. Hammel, J. Magn. Reson. 271, 15 (2016).

[39] R. Fischer, D. P. McNally, C. Reetz, G. G. T. Assumpção, T. Knief, Y. Lin, and C. A. Regal, New J. Phys. 21, 043049 (2019).

[40] J. Košata, O. Zilberberg, C. L. Degen, R. Chitra, and A. Eichler, Phys. Rev. Appl. 14, 014042 (2020).

[41] See Supplemental Material at http://link.aps.org/supplem ental/10.1103/PhysRevApplied.15.L021001 for additional setup descriptions, more measurement data, sample preparation descriptions, and planned scanning cavity and membrane upgrades.

[42] M. Heritier, A. Eichler, Y. Pan, U. Grob, I. Shorubalko, M. D. Krass, Y. Tao, and C. L. Degen, Nano Lett. 18, 1814 (2018).

[43] C. Reetz, R. Fischer, G. Assumpçao, D. McNally, P. Burns, J. Sankey, and C. Regal, Phys. Rev. Appl. 12, 044027 (2019).

[44] L. Catalini, Y. Tsaturyan, and A. Schliesser, Phys. Rev. Appl. 14, 014041 (2020).

[45] D. Rugar, H. J. Mamin, and P. Guethner, Appl. Phys. Lett. 55, 2588 (1989).

[46] S. Kuehn, R. F. Loring, and J. A. Marohn, Phys. Rev. Lett. 96, 156103 (2006).

[47] M. Kisiel, E. Gnecco, U. Gysin, L. Marot, S. Rast, and E. Meyer, Nat. Mater. 10, 119 (2011).

[48] B. C. Stipe, H. J. Mamin, T. D. Stowe, T. W. Kenny, and D. Rugar, Phys. Rev. Lett. 87, 096801 (2001).

[49] F. R. Braakman and M. Poggio, Nanotechnology 30, 332001 (2019).

[50] J. Labaziewicz, Y. Ge, D. R. Leibrandt, S. X. Wang, R. Shewmon, and I. L. Chuang, Phys. Rev. Lett. 101, 180602 (2008).

[51] J. M. Nichol, E. R. Hemesath, L. J. Lauhon, and R. Budakian, Phys. Rev. B 85, 054414 (2012).

[52] M.-H. Trinh, M. Odorico, L. Bellanger, M. Jacquemond, P. Parot, and J.-L. Pellequer, J. Mol. Recognit. 24, 503 (2011).

[53] J. Moser, J. Güttinger, A. Eichler, M. J. Esplandiu, D. E. Liu, M. I. Dykman, and A. Bachtold, Nat. Nanotechnol. 8, 493 (2013).

[54] S. L. de Bonis, C. Urgell, W. Yang, C. Samanta, A. Noury, J. Vergara-Cruz, Q. Dong, Y. Jin, and A. Bachtold, Nano Lett. 18, 5324 (2018).

[55] N. Rossi, F. R. Braakman, D. Cadeddu, D. Vasyukov, G. Tutuncuoglu, A. F. i Morral, and M. Poggio, Nat. Nanotechnol. 12, 150 (2017).

[56] L. M. de Lepinay, B. Pigeau, B. Besga, P. Vincent, P. Poncharal, and O. Arcizet, Nat. Nanotechnol. 12, 156 (2017).

[57] P. Sahafi, W. Rose, A. Jordan, B. Yager, M. Piscitelli, and R. Budakian, Nano Lett. 20, 218 (2019).

[58] Y. Tao, J. M. Boss, B. A. Moores, and C. L. Degen, Nat. Commun. 5, 3638 (2014).

[59] A. M. Jayich, J. C. Sankey, B. M. Zwickl, C. Yang, J. D. Thompson, S. M. Girvin, A. A. Clerk, F. Marquardt, and J. G. E. Harris, New J. Phys. 10, 095008 (2008).

[60] M. Brunelli, D. Malz, A. Schliesser, and A. Nunnenkamp, Phys. Rev. Res. 2, 023241 (2020).

[61] B. Gotsmann and H. Fuchs, Phys. Rev. Lett. 86, 2597 (2001).

[62] W. M. Dougherty, K. J. Bruland, J. L. Garbini, and J. A. Sidles, Meas. Sci. Technol. 7, 1733 (1996).

[63] E. W. Moore, S. Lee, S. A. Hickman, L. E. Harrell, and J. A. Marohn, Appl. Phys. Lett. 97, 044105 (2010).

[64] R. Hanson, L. P. Kouwenhoven, J. R. Petta, S. Tarucha, and L. M. K. Vandersypen, Rev. Mod. Phys. 79, 1217 (2007).

[65] K.-Y. Meng, A. S. Ahmed, M. Baćani, A.-O. Mandru, X. Zhao, N. Bagués, B. D. Esser, J. Flores, D. W. McComb, H. J. Hug, and F. Yang, Nano Lett. 19, 3169 (2019). 